\title{
Short-term active distribution network operation under uncertainty
}

\author{
Sébastien Mathieu, Damien Ernst \\ University of Liège \\ Liège, Belgium \\ smathieu,dernst@uliege.be
}

\author{
Quentin Gemine \\ Blacklight Analytics \\ Liège, Belgium \\ qgemine@blacklight-analytics.com
}

\begin{abstract}
Electrical distribution systems need to integrate more and more renewable energy generation in their network. Since networks cannot be quickly upgraded at a low cost, new generators are connected to the network under non-firm access contracts. These contracts allow distribution system operators to specify dynamic access limits according to a given regulatory policy, e.g. "last-in, first-out" or a similar policy. Due to operational delays, access limits must be communicated before realtime, e.g. ten minutes ahead. This paper presents an operational method to compute these dynamic access limits using correlated probabilistic forecasts of power consumption and production processes. The method is illustrated on a test-case based on real data where no additional production would be allowed under firm access. Results show that the method allows to safely integrate additional production capacity while limiting congestion events, provided that efficient probabilistic forecasts able to anticipate sudden and important changes are available.
\end{abstract}

\section{INTRODUCTION}

Distribution System Operators (DSO) aim for active network management strategies to address congestion problems using short-term decision-making policies [1]. Many actions can be taken beforehand such as network reconfiguration, tap-changer adjustments, etc. Despite all these actions, DSOs struggle to integrate the increasing amount of distributed generation. This increased production creates congestion problems in critical parts of distribution networks that need to be addressed. To alleviate congestion problems, the European regulatory policy allows DSOs to limit the access of generators to their networks. This access is traditionally formalized by firm-access contracts defining access limits re-evaluated yearly at best. Computing these long-term limits is not straightforward. The paper [2] provides a pragmatic approach to compute such limits which relies on tools that are routinely used in distribution system planning and operation. Firm access limits to the network are however inefficient since they are computed on worst-case scenarios over large time periods. The paper [3] shows, via a test case, that setting dynamic access limits can increase the production of generators by $55 \%$, considering uncertainty on both production and consumption. This result is obtained with a technical optimum curtailment policy which does not consider regulatory constraints. Regulators often impose "last-in, first-out" (LIFO) policies, i.e. the last generator installed is the first to be limited. Alternative regulatory policies are investigated in the literature, see paper [4]. Examining the choice of the most appropriate regulatory policy is outside the scope of this paper.

Given a regulatory policy, this paper focuses on providing a practical method to compute dynamic access limits updated on a regular basis, e.g. every five minutes. Owing to technical constraints and operational delays, access limits must be communicated before real-time, e.g. ten minutes ahead. The limitsetting decision process must therefore be based on forecasts of the consumption and production. Truly optimal decisions and risk hedging decisions are only possible with the adoption of probabilistic forecasts [5]. For instance, prediction error of very-short-term wind power forecasts can reach up to $30 \%$. Most probabilistic forecasting techniques provide Gaussian Probability Density Functions (PDF). Gaussian distribution cannot accurately model the problem tackled in this paper. In particular, the energy production of a limited generator cannot be accurately modeled by a Gaussian PDF. This problem is addressed in the method proposed in this paper. The method is (i) able to define dynamic access limits for generators ensuring the safety of the network, (ii) based on non-Gaussian correlated PDF, (iii) following a given regulatory curtailment policy, (iv) in a computationally tractable implementation. Note that providing generator limits differs from a dispatching procedure since regulation does not allow providing set-points and only allows to provide production limits if there is an anticipated congestion risk.

The paper is structured as follows. The relevant literature is reviewed in Section II. Section III mathematically defines the problem of determining dynamic access limits to generators under uncertainty. One practical method to solve this problem is described in Section IV where generators must be curtailed following a specific order. Simulation results are presented in Section V. Finally, Section VI concludes.

\section{LITERATURE REVIEW}

To our knowledge, the current state of the literature does not cover this specific case that is currently faced by some DSOs. The definition of long-term non-firm access limits is investigated in [6] by applying optimal power flow to extract maximum energy from available renewable resources whilst using curtailment of generation to maintain the network within thermal and voltage limits at an hourly resolution. The paper mention that sub-hourly resource estimation would be 
necessary to improve estimates of operation and curtailment and even greater levels of detail are required for real-time control [6]. Another example of non-firm access management in planning and operation is presented in [7]. The operation phase is based on a deterministic optimal power flow. Results show a significant increase in capacity allocation and energy produced when comparing the firm and non-firm accesses. The operation considering switching off generators in radial distribution networks is formulated as a mixed integer quadratically constrained problem in the paper [8]. A risk-limiting optimal power flow is presented in the paper [9] where renewable generation is redispatched to compensate deviations between the actual and predicted wind power generations. The solution is based on an off-line Monte-Carlo sampling coupled with regular optimal power flow, allowing PDF of any type. The decision phase is an approximation based on the off-line sampling. The paper [10] presents a controller which ensures that the power at the high-voltage/medium-voltage interface remains within specified active and reactive power limits. The algorithm uses a linear power flow and assumes that power consumptions and productions are Gaussian variables.

\section{GENERIC PROBLEM STATEMENT}

As is a common practice in power system operation, time is divided into time periods hereby of length $\Delta t$, e.g. five minutes. Starting from a time $t$, the time line of the decision problem faced by the DSO is depicted in Figure 1. The DSO has one time period to perform its forecasts and computations. At the beginning of the next period, in $t+\Delta t$ the access limits are communicated and generators have one time period within which to comply. Therefore, the operational period where limits are effectively applied ranges from $t+2 \Delta t$ to $t+3 \Delta t$. Thereafter, time indexes are omitted to simplify notations.

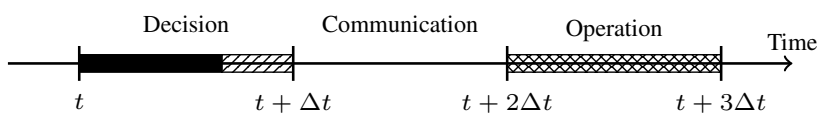

Fig. 1: Time line of the operational decision problem.

In time $t$, a topology is extracted from the operational state of the distribution network. This topology contains a set of constrained asset $\mathcal{A}$ such as critical lines, transformers, etc. The electrical power flowing through one asset $a \in \mathcal{A}$ must be lower than its maximum tolerated power, $p_{a}^{\max }$. To satisfy this condition, the DSO controls the maximum production of a set of generators $\mathcal{G}$. An example of network topology illustrating the notations is given in Figure 2.

Each generator $g \in \mathcal{G}$ may receive an access limit $\bar{p}_{g}$ lower than or equal to its maximum installed capacity $p_{g}^{\max }$. The complete set of access limits is denoted by $\overline{\mathcal{P}}=\left\{\bar{p}_{g} \mid \forall g \in \mathcal{G}\right\}$ and the unrestrained one by $\mathcal{P}^{\max }=\left\{p_{g}^{\max } \mid \forall g \in \mathcal{G}\right\}$. Setting these limits influences the power flowing through each asset $a$. In time $t$, the DSO only has access to forecasts of the power flow. A forecast for an asset $a$ is given by a PDF $f_{a}(p \mid \overline{\mathcal{P}})$ providing the probability of a power flow of $p$. The network is considered safe if the congestion risk of

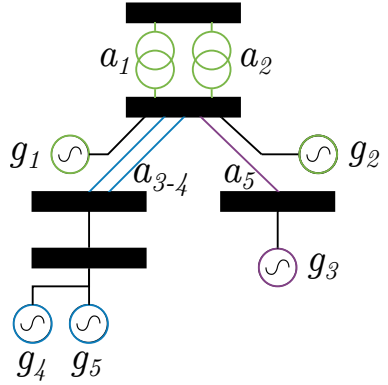

Fig. 2: Schematic representation of a network with constrained assets indexed by $a$ and generators by $g$.

each asset $a \in \mathcal{A}, r_{a}(\overline{\mathcal{P}})$, is less than a given maximum tolerated congestion risk, $R_{a}$, e.g. $1 \%$. The DSO objective is to minimize the expected curtailment such that the network is safe. The regulatory policy imposes constraints on how access limits can be specified. In order to be generic, the set of valid combination of access limits is denoted by $\mathcal{Z}$ such that a valid solution satisfies $\overline{\mathcal{P}} \in \mathcal{Z}$. In most cases, the set of constraints $\mathcal{Z}$ imposes a specific curtailment order for the generators, such as the LIFO policy. The Walloon regulator imposes a LIFO variant [11] which is at the origin of this paper.

Using the previous notation, the generic stochastic decision problem (1) is given by equations (1a)-(1d).

$$
\min \sum_{g \in G} \int_{\bar{p}_{g}}^{+\infty}\left(p-\bar{p}_{g}\right) f_{g}\left(p \mid \mathcal{P}^{\max }\right) d p
$$

subject to

$$
\begin{array}{ll}
r_{a}(\overline{\mathcal{P}})=\int_{p_{a}^{\max }}^{+\infty} f_{a}(p \mid \overline{\mathcal{P}}) d p \leq R_{a} & \forall a \in \mathcal{A} \\
p_{g}^{\min } \leq \bar{p}_{g} \leq p_{g}^{\max } & \forall g \in \mathcal{G} \\
\overline{\mathcal{P}}=\left\{\bar{p}_{g} \mid \forall g \in \mathcal{G}\right\} \subset \mathcal{Z} &
\end{array}
$$

Decision problem (1) relies on the tail of the computed distributions, in particular due to the expression of the risk tolerance in equation (1b). It is therefore important to correctly model the distribution tails. This paper assumes that setting a limit clips the PDF tail and shifts the clipped part at the limit such that the risk of a generator producing in excess of its access limit is null. A visual example of such clipping is given in Figure 3a. Applying a limit on a generator, i.e. clipping its production PDF, modify the PDF of the power flowing through the transformer connecting the distribution network to the transmission network. An example of modified PDF of the power flowing through the transformer is given in Figure $3 b$, where the peak that appears on the right is due to the clipping of a generator PDF.

\section{RESOLUTION PROCEDURE}

This section details a resolution procedure for problem(1) in a case where regulation imposes a LIFO policy, denoted by $\mathcal{Z}^{L I F O}$, in a radial distribution network. Imposing a curtailment order requires that, to limit a generator, all access limits before this one in the curtailment order must be set 


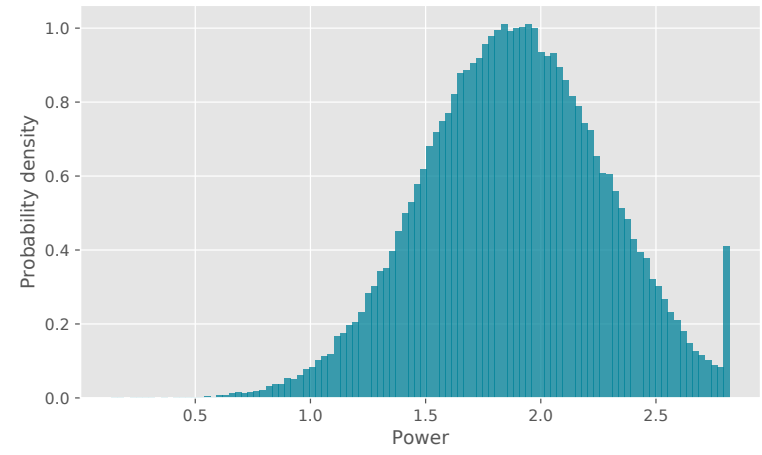

(a) Production PDF of a wind turbine limited at 2.8 MW.

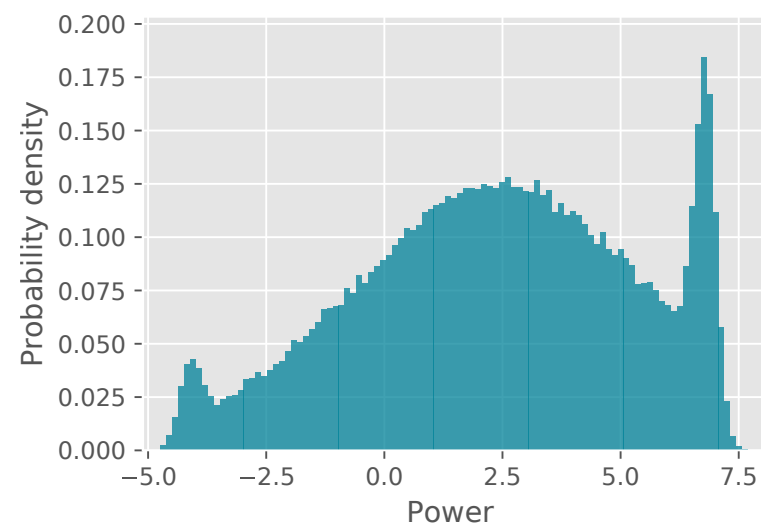

(b) PDF of the power flowing through a transformer.

Fig. 3: PDF examples of $f_{g}(p \mid \overline{\mathcal{P}})$ and $f_{a}(p \mid \overline{\mathcal{P}})$.

equal to zero or their technical minimum. Such a curtailment order is a common regulatory practice. A real-life example is the curtailment policy imposed by the Walloon regulator [11] which corresponds to a LIFO policy variant. The implementation of this policy only requires minor adjustments to the generic procedure that is presented hereafter.

The resolution process is divided into three phases: generation of a congestion risk model, forecasting of the power production PDF, computation of the access limits. These steps are detailed in Sections IV-A, IV-B and IV-C.

\section{A. Congestion risk model}

The resolution procedure starts by interpreting the topology and configuration of the distribution network as a directed acyclic graph. In practice, most distribution networks are operated radially, i.e. no loop exists except for the one created by lines, cables and transformers that are operated in parallel. The leaves of the resulting graph are power injection sources, where consumption is considered as negative injection. The set of sources is denoted $\mathcal{S}$ and includes all generators whose limits can be set. The power injection of a source $s \in \mathcal{S}$ is modeled as a random variable $X_{s}$ following the $\operatorname{PDF} f_{s}(p \mid \overline{\mathcal{P}})$.

To each node $n$ of the graph is associated a set of assets $\mathcal{A}_{n} \subset \mathcal{A}$. The flow going through each node $n \in \mathcal{N}$ is modeled as the sum of power injections of all downstream sources $\mathcal{D}_{n}$ :

$$
X_{n}=\sum_{s \in \mathcal{D}_{n}} X_{s} .
$$

This equation assumes that network losses can be neglected, therefore leading to conservative power limits with respect to network congestions caused by production excesses. The power flow going through an asset $a \in \mathcal{A}_{n}$ of a node $n$, is considered to be a share $w_{a}$ of the flow in the node:

$$
X_{a}=w_{a} \cdot X_{n} .
$$

For instance, two identical parallel transformers would get coefficients $w_{a}=1 / 2$. The random variable $X_{a}$ is then defined by the PDF $f_{a}(p \mid \overline{\mathcal{P}})$ which allows for computing of the congestion risk $r_{a}(\overline{\mathcal{P}})$ as defined in Equation (1b).

Computing the sum (2) analytically is not straightforward since the PDF expressing $X_{s}$ may be non-Gaussian and correlated to other sources. The implementation of the procedure uses PDF samples to compute this sum, making the computation of $r_{a}(\overline{\mathcal{P}})$ straightforward, given $f_{s}(p \mid \overline{\mathcal{P}})$. The source PDFs are modeled using a multivariate Gaussian sampling based on forecasted mean power injection $\boldsymbol{\mu}$ and a covariance matrix $\boldsymbol{\Sigma}$, obtained as explained in the following Section IV-B. The forecasted power injection samples for a generator $g$ are modified to consider generator capacities by clipping the samples below zero and above the access limit $\bar{p}_{g}$.

\section{B. Forecasting}

The forecasting procedure takes historical measurements of the network as inputs and outputs a vector of forecasted power injection means $\boldsymbol{\mu}$ and a covariance matrix $\boldsymbol{\Sigma}$ used for multivariate Gaussian distribution sampling (cf. Section IV-A).

The forecasting procedure starts with recovering historical power injection time series for each modeled source $s \in \mathcal{S}$ of the network. This step is obvious if a sensor is associated with the source, which is often the case for generators. Consumption sources are rarely monitored at a minute resolution. Their historical time series must be inferred from other measurements, usually placed before the breaker associated to the feeder where the load is connected. The residual load consumption can be inferred by subtracting from the latter measurement the sum of power measurements from downstream generators.

The forecasting procedure uses the historical power injection time series in two steps: (i) individual forecasting of each source, (ii) estimation of a covariance matrix. The individual forecasting is performed using an independent Gaussian process regression for each source time series that provides a forecasted mean power injections vector, denoted $\boldsymbol{\mu}$, and a power injection variances vector $\sigma^{2}$. More details about this step are given in Appendix VII. The second step of covariance estimation can be performed using any suitable technique, such as the Minimum Covariance Determinant estimates used in the results section. The resulting initial covariance matrix, denoted $\boldsymbol{\Sigma}^{0}$, contains diagonal terms which are different from the one forecasted by Gaussian process regression, $\rho$. A scaled covariance matrix considering the individual forecast is given by $\boldsymbol{\Sigma}=\boldsymbol{W} \boldsymbol{\Sigma}^{0} \boldsymbol{W}^{T}$ where $\boldsymbol{W}$ is a diagonal matrix whose elements are given by the vector $\sigma / \sqrt{\operatorname{diag}\left(\Sigma^{0}\right)}$. 


\section{Access limits computation}

Since the access limits must be set following a curtailment order $\mathcal{Z}^{L I F O}$, problem (1) can be solved by curtailing generators one by one until the congestion risk becomes tolerable. Only the last curtailed generator in that order receives a limit greater than its technical minimum. Suppose this generator $g$ is identified and let the set of access limits without $g$ be:

$$
\overline{\mathcal{P}}_{g}=\left\{\bar{p}_{g^{\prime}} \mid g^{\prime} \in \mathcal{G} \backslash\{g\}\right\} .
$$

The limits $\bar{p}_{g^{\prime}}$ in this set represent either the technical minimum of the generators coming before $g$ in the curtailment order or the maximum possible power for other generators. This limit of the last curtailed generator, $\bar{p}_{g}$, can be computed by any root-finding algorithm on $r_{a}\left(\overline{\mathcal{P}}_{g}\right)-R_{a}$, for instance the secant method, with some safeguards to account for the nonsmoothness caused by distribution clipping. The procedure to obtain the maximum access limits is described in Algorithm 1.

Require: $\left\{r_{a}(\overline{\mathcal{P}}) \mid a \in \mathcal{A}\right\}$, the set of congestion risk functions 1: $\overline{\mathcal{P}} \leftarrow \mathcal{P}^{\max }$

$$
\begin{aligned}
& \text { for each } g \in \mathcal{G} \text { following } \mathcal{Z}^{L I F O} \text { do } \\
& \text { for each } a \in \mathcal{A} \text { do } \\
& \text { if } \exists p_{g} \in\left[p_{g}^{\min }, \bar{p}_{g}\right]: R_{a}-r_{a}\left(\left\{p_{g}\right\} \cup \overline{\mathcal{P}}_{g}\right)=0 \text { then } \\
& \bar{p}_{g} \leftarrow p_{g}: R_{a}-r_{a}\left(\left\{p_{g}\right\} \cup \overline{\mathcal{P}}_{g}\right)=0 \\
& \text { else } \\
& \bar{p}_{g} \leftarrow p_{g}^{\min } \\
& \text { end if } \\
& \text { end for } \\
& \text { end for }
\end{aligned}
$$

Algorithm 1: Decision algorithm to obtain the maximum access limits following the curtailment order $\mathcal{Z}^{L I F O}$.

\section{RESUlts}

This section shows the algorithm performance on an academic network. Results are obtained from a Python 3 code, using scikit-learn for Gaussian process regression and covariance estimation [12]. Each PDF is represented by one hundred thousand sampling points.

\section{A. Performance metrics}

In a simulation, the maximum production of a curtailed generator $g, p_{g}$, and the power flow without curtailments going through an asset $a, p_{a}$, are known and may be used to build performance metrics. Given an asset $a \in \mathcal{A}$ and $\mathcal{G}_{a}$, the set of generators downstream with respect to asset $a$, the total curtailed power to ensure the safety of asset $a$ is given by

$$
c_{a}=\sum_{g \in \mathcal{G}_{a}} \max \left\{0, p_{g}-\bar{p}_{g}\right\} .
$$

The primary objective of the decision problem is to set sufficiently low access limits to ensure safe network operation. The under-curtailment metric aims at quantifying the amount of curtailment lacking from the decision strategy, leaving the network unsafe. This metric is defined by

$$
U C_{a}(\overline{\mathcal{P}})=\max \left\{0,\left(p_{a}-c_{a}\right)-p_{a}^{\max }\right\} .
$$

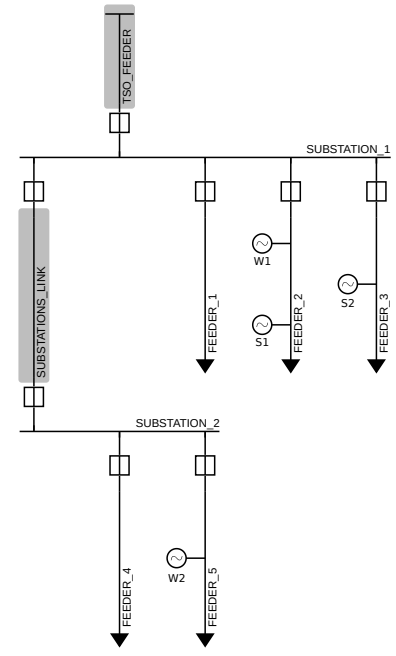

Fig. 4: Test-case network topology.

Obtaining the minimum under-curtailment score is easily achieved with a strategy setting each access limit to their technical minimum. It is therefore important to define a second performance metric quantifying the over-curtailment with respect to an optimal curtailment case where the future is known. The over-curtailment power is defined by the metric

$$
O C_{a}(\overline{\mathcal{P}})=\max \left\{0, c_{a}-\max \left\{0, p_{a}-p_{a}^{\max }\right\}\right\} .
$$

\section{B. Test case}

Figure 4 presents the medium-voltage network considered in this test case. A main substation is connected to the transmission network by a feeder named TSO_FEEDER. A secondary substation is connected to the first by a feeder named SUBSTATIONS_LINK. In this test case, the injection limit set to these two constrained feeders is $2 \mathrm{MW}$. In practice the value of these limits may be given by transmission system operator requirements, voltage stability, etc. The network contains four generators whose characteristics are given in Table I. The power injection associated to feeders TSO_EEEDER and SUBSTATIONS_LINK are historical time series of -3.13 MW and -2.79 MW means and 3.07 MW and 0.45 MW standard deviations. With these time series, the total power injection without the four generators goes over $2 \mathrm{MW}$ in several occasions. Therefore, with the given feeder injection limits no generators would be granted access to the network.

TABLE I: Test case generator parameters.

\begin{tabular}{c|c|c|c} 
Id & Type & Capacity & Priority \\
\hline W1 & Wind & $10 \mathrm{MW}$ & 1 \\
W2 & Wind & $5 \mathrm{MW}$ & 3 \\
S1 & Solar & $4 \mathrm{MW}$ & 4 \\
S2 & Solar & $8 \mathrm{MW}$ & 2
\end{tabular}

Decisions are taken each five minutes over four days based on real-life measurements at one-minute resolution, available for the power flow going out of each generator, each access point and through the two constrained feeders. The total simulation takes less than two hours on a standard computer 
with the large majority of the computation time dedicated to the forecasting process. The unconstrained power flow in TSO_FEEDER is represented by the gray line in Figure 5. This figure shows the forecasted mean values by the blue line, the variance represented by the inner blue area, and the $0.25 \%-99.75 \%$ quantiles represented by the outer blue areas. Without access limits, the power flow would be greater than the tolerated limit, given by the red line, $28 \%$ of the time.

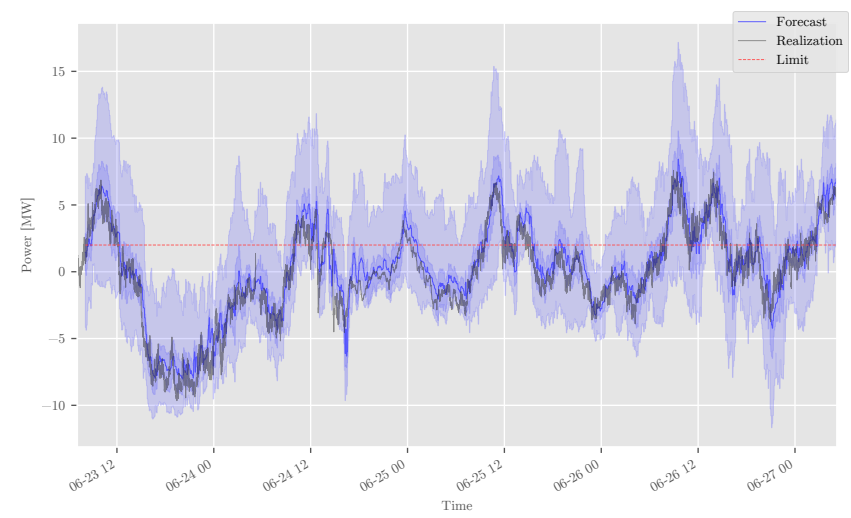

Fig. 5: Forecasts and non-limited realization at the connection to the transmission network.

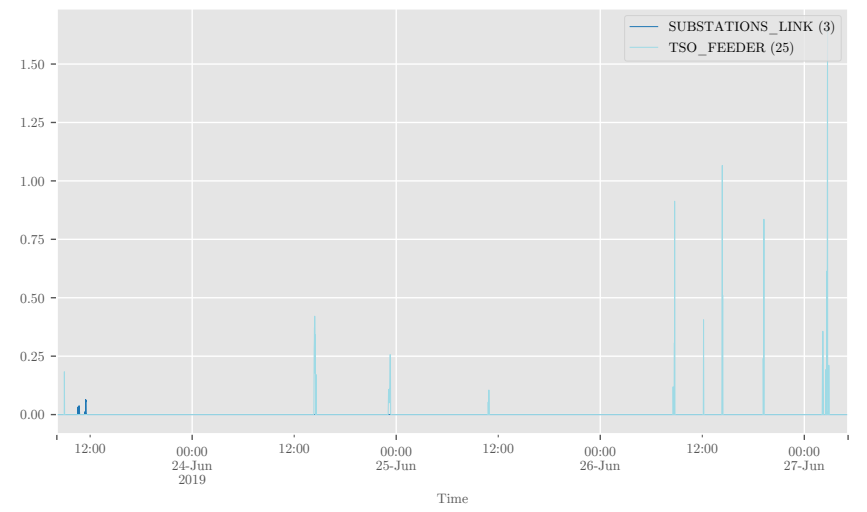

(a) Under-curtailment power, $U C_{a}(\overline{\mathcal{P}})$, in MW.

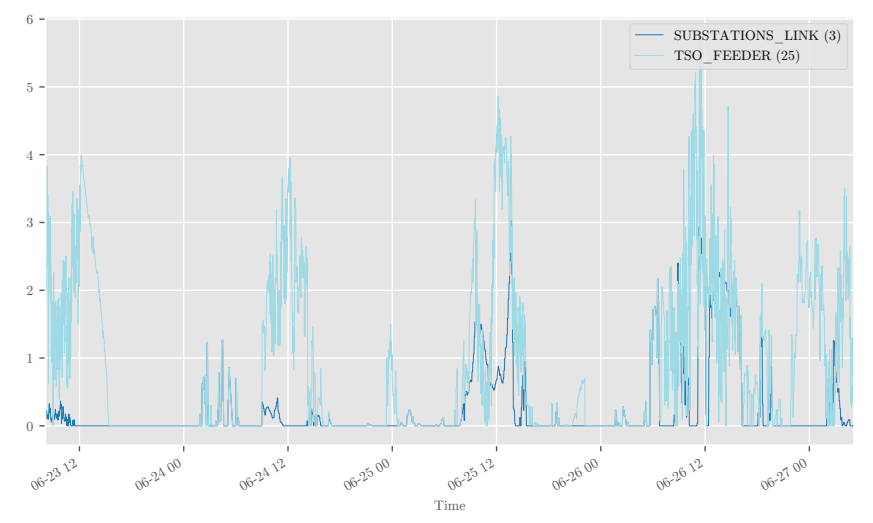

(b) Over-curtailment power, $O C_{a}(\overline{\mathcal{P}})$, in MW.

Fig. 6: Performance metrics for the two constrained assets.

The algorithm efficiently reduces the power flow to below the tolerance of the two constrained assets. Figure 6a shows that the tolerance of asset TSO_FEEDER is violated on rare occasions. There are 78 minutes of under-curtailment over the simulation period with a maximum of 16 consecutive minutes. Most of these cases occur when realization is above the forecast at the maximum tolerated risk. These 78 minutes correspond to a risk of $1.36 \%$, i.e. of the same order of magnitude that the algorithm input $R_{a}=1 \%$. The power excess during these events ranged from $5 \mathrm{~kW}$ to $1.65 \mathrm{MW}$. Obviously, these results are very dependent on forecast quality. Figure $6 \mathrm{~b}$ shows the over-curtailment as defined in Equation (7). This figure clearly shows that actions are taken only when total power is close to the constrained asset-tolerated flow.

\section{Risk tolerance sensitivity analysis}

This section studies how the risk tolerance influences undercurtailment and over-curtailment performance metrics on the TSO_EEEDER asset of the test case represented in Figure 4. The test case is a particularly stressful one since the largest production increase in a five-minute period corresponds to 4 MW, $200 \%$ of the feeder injection limits, while the standard deviation of the load is of the same order of magnitude than feeder limits.

Table II summarize this sensitivity analysis for risk tolerances $R_{a}$ from $0.01 \%$ to $1 \%$. Several observations can be drawn from this table. As expected, the maximum undercurtailment power reduces as the risk tolerance decreases. With perfect probabilistic forecasts, the percentage of time where under-curtailment events occur should in principle be equal, in expectation, to the risk $R_{a}$. However, the events reported in the table seem to overestimate their corresponding values of $R_{a}$. As a result, the system is operated with a higher degree of risk than the one determined by the parameter $R_{a}$. The estimated additional risk is for example $0.38 \%$ when $R_{a}$ is equal to $1 \%$ and $0.42 \%$ when $R_{a}$ is equal to $0.01 \%$. Further analyses not reported in this paper have shown that this additional risk is due to extreme events defined as particularly abrupt increases in production that could not be forecasted by the Gaussian process. As for the over-curtailments, they lead to an energy loss that grows as the risk level $R_{a}$ decreases, which is also expected. With the smallest value of $R_{a}$, the over-curtailment leads to $93.98 \mathrm{MWh}$ of wasted energy. This corresponds to $20 \%$ percent of the total energy that would be produced by the four generators without any curtailment.

\section{CONCLUSION}

This paper formalizes the operational problem faced by a DSO in setting short-term access limits under uncertainty following a given curtailment regulatory policy. One resolution method is proposed in the case of a radial distribution network where the regulatory policy imposes a curtailment order of the generators. The proposed method relies on clipped multivariate Gaussian sampling to consider correlation between the various production and consumption sources of the network. The method is illustrated on a challenging distribution network test case where no additional production would be allowed under firm access. Results on this test case show that the method 
TABLE II: Risk tolerance sensitivity results of under-curtailment, $U C_{a}(\overline{\mathcal{P}})$, and over-curtailment, $O C_{a}(\overline{\mathcal{P}})$, on the TSO_FEEDER asset of the network represented in Figure 4.

\begin{tabular}{|c|c|c|c|c|c|c|c|}
\hline \multirow[b]{2}{*}{ Risk - $R_{a}[\%]$} & \multicolumn{3}{|c|}{$U C_{a}(\overline{\mathcal{P}})$} & \multicolumn{2}{|c|}{$U C_{a}(\overline{\mathcal{P}})$ duration } & \multicolumn{2}{|c|}{$O C_{a}(\overline{\mathcal{P}})$} \\
\hline & Events [\%] & Energy [MWh] & $\operatorname{Max}[\mathrm{MW}]$ & Mean [min] & $\operatorname{Max}[\min ]$ & Energy [MWh] & $\operatorname{Max}[\mathrm{MW}]$ \\
\hline 0.01 & 0.43 & 0.14 & 1.44 & 3.33 & 6.00 & 93.98 & 6.61 \\
\hline 0.05 & 0.79 & 0.21 & 1.65 & 4.56 & 15.00 & 88.85 & 6.62 \\
\hline 0.10 & 0.88 & 0.25 & 1.65 & 4.60 & 16.00 & 85.48 & 5.86 \\
\hline 0.25 & 1.00 & 0.31 & 1.65 & 4.08 & 16.00 & 80.37 & 5.86 \\
\hline 0.50 & 1.15 & 0.36 & 1.65 & 4.25 & 16.00 & 76.22 & 5.50 \\
\hline 1.00 & 1.38 & 0.41 & 1.65 & 4.36 & 16.00 & 71.44 & 5.30 \\
\hline
\end{tabular}

reduces congestion events below $1 \%$, compared to $28 \%$ if the production was not limited.

To obtain smaller congestion risks, the method would require more accurate probabilistic forecasts of extreme events. In this application, abrupt production increases are of the upmost importance since they are likely to lead to congestion in the network. This often happens when looking at oneminute resolution time series of wind-power production which includes nearly an instantaneous increase by half of the production unit capacity. Other improvements of this technique could be considered, such as incorporating network losses or handling meshed networks, relying on the stochastic optimal power flow literature. The operational method described in this paper could also be used to perform quantitative analyses of regulatory curtailment policies. Moreover, additional research to find an optimal regulatory policy would be of interest, yet not straightforward since it should aim at compromising between fairness and technical optimum.

\section{APPENDIX - GAUSSIAN PROCESS REgRESSION}

The set of time stamps used as history to predict the production and the consumption in time stamp $t$ is denoted by $\mathcal{H}_{t}$. Since Gaussian process regression uses the complete set of inputs to perform the prediction, the history $\mathcal{H}_{t}$ must be limited. In practice, the history typically covers the last two hours with a point every minute. Note that Gaussian process regression comfortably tolerates gaps in the input. The input for training the Gaussian process regressor is, for each time $\tau \in \mathcal{H}$, the time difference between the target time and the measurement in minutes $\tau-t$ and the historical value $p_{\tau}$. The kernel functions used for regression are the constant kernel, the white kernel and the exponential sinus squared kernel, respectively denoted $k^{c}\left(\mathbf{x}, \mathbf{x}^{\prime}\right), k^{w}\left(\mathbf{x}, \mathbf{x}^{\prime}\right), k^{s}\left(\mathbf{x}, \mathbf{x}^{\prime}\right)$. The total kernel used for regression is

$$
k\left(\mathbf{x}, \mathbf{x}^{\prime}\right)=k^{c}\left(\mathbf{x}, \mathbf{x}^{\prime}\right) k^{r}\left(\mathbf{x}, \mathbf{x}^{\prime}\right)+k^{s}\left(\mathbf{x}, \mathbf{x}^{\prime}\right) .
$$

The Gaussian process regression is applied to each source $s \in$ $\mathcal{S}$ in time step $t$ to obtain the PDF forecast $\mathcal{N}\left(\mu_{s}, \sigma_{s}\right)$ for the period $[t+2 \Delta t, t+3 \Delta t]$.

\section{REFERENCES}

[1] Q. Gemine, D. Ernst, and B. Cornélusse, "Active network management for electrical distribution systems: Problem formulation, benchmark, and approximate solution," Optimization and Engineering, vol. 18, no. 3, pp. 587-629, 2017.
[2] B. Cornélusse, D. Vangulick, M. Glavic, and D. Ernst, "Global capacity announcement of electrical distribution systems: A pragmatic approach," Sustainable Energy, Grids and Networks, vol. 4, pp. 43-53, 2015.

[3] S. Mathieu, D. Ernst, and B. Cornélusse, "Agent-based analysis of dynamic access ranges to the distribution network," in 2016 IEEE PES Innovative Smart Grid Technologies Conference Europe (ISGT-Europe), IEEE, 2016, pp. 1-6.

[4] L. Kane and G. W. Ault, "Evaluation of wind power curtailment in active network management schemes," IEEE Transactions on Power Systems, vol. 30, no. 2, pp. 672-679, 2014.

[5] R. Bessa, C. Möhrlen, V. Fundel, M. Siefert, J. Browell, S. Haglund El Gaidi, B.-M. Hodge, U. Cali, and G. Kariniotakis, "Towards improved understanding of the applicability of uncertainty forecasts in the electric power industry," Energies, vol. 10, no. 9, p. 1402, 2017.

[6] T. Boehme, G. P. Harrison, and A. R. Wallace, "Assessment of distribution network limits for non-firm connection of renewable generation," IET renewable power generation, vol. 4, no. 1, pp. 64-74, 2010.

[7] M. Džamarija, M. Bakhtvar, and A. Keane, "Operational characteristics of non-firm wind generation in distribution networks," in 2012 IEEE Power and Energy Society General Meeting, IEEE, 2012, pp. 1-8.

[8] I. Bilibin and F. Capitanescu, "Contributions to thermal constraints management in radial active distribution systems," Electric Power Systems Research, vol. 111, pp. 169-176, 2014.

[9] S.-Y. Lin and A.-C. Lin, "RLOPF (risk-limiting optimal power flow) for systems with high penetration of wind power," Energy, vol. 71, pp. 49-61, 2014.

[10] J. Buire, F. Colas, J.-Y. Dieulot, and X. Guillaud, "Stochastic optimization of PQ powers at the interface between distribution and transmission grids," Energies, vol. 12, no. 21, p. 4057, 2019.

[11] Service public de Wallonie, Arrêté du gouvernement wallon relatif à l'analyse coût-bénéfice et aux modalités de calcul et de mise en ouvre de la compensation financière, [2016/206014], 2016.

[12] Scikit-learn: Machine learning in Python, https://scikitlearn.org/, 2020. 